\title{
SCIDic
}

\author{
International Journal of Dentistry and Oral Science (IJDOS) \\ ISSN: $2377-8075$
}

\section{Ignored Amalgam Pigmentation Leading to Discomfort upon Denture wearing: A Case Report}

Case Report

Qing Sheng Tan ${ }^{1}$, Anitha Krishnan Pandarathodiyil ${ }^{2}$, Jacob John ${ }^{1}$, Karthick Sekar ${ }^{1}$, PL Ranganayakidevi S Palaniappan ${ }^{1}$, Anand Ramanathan ${ }^{1,3}$

${ }^{1}$ Faculty of Dentistry, University of Malaya, 50603, Kuala Lumpur, Malaysia.

${ }^{2}$ Faculty of Dentistry, SEGi Univeristy, Jalan Teknologi, Kota Damansara, 47810, Petaling Jaya, Selangor, Malaysia.

${ }^{3}$ Oral Cancer Research \& Coordinating Center, Faculty of Dentistry, University of Malaya, 50603, Kuala Lumpur, Malaysia.

\section{Abstract}

Pigmented oral lesions are commonly encountered in clinical dental practice. They range from physiologic changes to manifestation of systemic diseases or neoplasms, sometimes posing diagnostic challenges. In this article, a case of a 73-year-old male with a pigmented oral macule on his lower left alveolar ridge, which was ignored initially, is reported. The patient's complaint included an ill-fitting denture causing discomfort, arising due to the tenderness over the pigmented lesion. Investigations including biopsy, histopathology, and energy dispersive X-ray analysis (EDX) were carried out to determine the definitive diagnosis of amalgam tattoo. The clinical features, etiology, histopathology, and management of this case are briefly discussed. After surgical intervention to remove this pigmentation, the healing was uneventful without tenderness upon palpation and proceed to construction of removable prosthesis. Nevertheless, the importance of thorough investigation of an ignored amalgam tattoo in edentulous areas, before fabricating dentures form the essence of this case report.

Keywords: Oral Pigmentation; Amalgam Tattoo; Tenderness; Alveolar Ridge; Denture Fabrication.

\section{Introduction}

Pigmentations of the oral mucosal tissues are relatively common findings in clinicalpractice. It is the process of incorporation of endogenous or exogenous pigments withintissues. These could be manifestations of disease processes, anatomic or physiologicvariations, or innocuous lesions accidentally deposited into the tissues. While considering exogenous pigmentations of the oral mucosa, the usual suspect is the amalgam tattoo, with graphite next in line, although way less common [1]. It is reported to be caused when tiny specks of dental amalgam particles are inadvertently implanted into oral soft tissues during dental procedures. We, herewith, discuss a case of ignored pigmented lesion leading to an ill-fitting denture and tenderness upon palpation which presented in an unusual manner than reported ever before in the literature as amalgam tattoo is normally asymptomatic and harmless most of the time.

\section{Case Presentation}

A 73-year-old retired Chinese male tax accountant presented at the dental clinic, requesting for a new set of dentures. The patient was edentulous and gave a history of wearing the current complete dentures for past 6 months. He gave a medical history of depression and hypercholesterolemia, both of which were under control with medication and routine follow-up. Patient takes Amisulphide $400 \mathrm{mg}$ twice a day, IV Paliperidone 100mg monthly, Clozapine 50mg per day, Artane $2 \mathrm{mg}$ three times a day, Aspirin $100 \mathrm{mg}$ and Simvastatin 40mg per day. Dental history revealed that the last extraction was done about 6 years ago, and dental caries was the reason for most of the extractions. Patient was aware that he had multiple restored teeth but could not recall when or how many he had or what material was used for the restoration. He is currently wearing his second set of dentures. The first set of dentures was fabricated in 2015. According to the patient the stability, retention, aesthetic, phonetic, and masticatory functions of the previous denture were in acceptable condition. Six months ago, he requested for a spare set of dentures in case of loss or breakage of the denture. Patient was satisfied with the new set of dentures and wore them throughout the day, except while sleeping. Following up with the patient's complaint, he revealed that he has been experiencing some discomfort while chewing on the

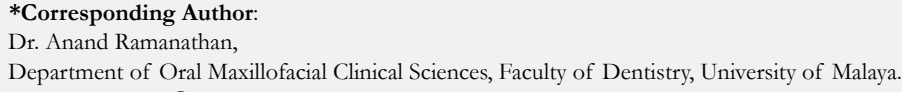

Copyright: Anand Ramanathan 2021 . This is an open-access article distributed under the terms of the Creative Commons Attribution License, which permits unrestricted use, distribution and reproduction in any medium, provided the original author and source are credited. 
left side of his lower jaw. The discomfort was present from the time he started wearing the new set of dentures, for which he followed-up with the previous dentist who constructed the dentures, for denture adjustments. However, even after the denture adjustments, the patient was not relieved of his discomfort and hence the current consultation with us for the ill-fitting denture.

\section{Clinical Findings}

On intraoral examination, he was confirmed edentulous. The upper and lower ridges were ovoid in shape and well formed. The mucosal consistency was normal. There was no soreness or redness found on the mucosa. The oral hygiene was well maintained. On the left lower alveolar ridge, a black pigmented, diffuse macule measuring about $4 \mathrm{~mm}$ in diameter, was noticed (Figure 1a). Upon palpation of the pigmented area, there was tenderness. The patient felt relief upon removal of the pressure.

\section{Diagnostic Assessment and Management}

Since the pigmented lesion showed signs of tenderness upon palpation, a decision was made to investigate further in order to confirm the diagnosis and eliminate the possibility of malignant melanoma. Therefore, the patient was referred to an Oral Medicine specialist. After obtaining the required informed consent from the patient, a biopsy was performed, and the tissue sent for histopathological examination. The tissue was cut into two pieces and processed. The blade of the microtome broke while trying to section one of the tissues. Careful examination of the wax block revealed a $3 \mathrm{~mm}$ piece of metallic structure that was embedded within the tissue (Figure 1b). Therefore, the second piece of tissue was sectioned and stained with hematoxylin and eosin. Histopathological examination revealed surface parakeratinized stratified squamous epithelium with a cleft artifact in the center, which was corresponding to the metal fragment present in the other piece of tissue that could not be sectioned. There were numerous small spots of black pigmentations were strewn in the epithelium and the connective tissue (Figure 1c). A diagnosis of amalgam tattoo with embedded metallic content was made. Review of the previous dental panoramic tomography (DPT) revealed a speck of radiopacity, of about 3 to $4 \mathrm{~mm}$ diameter corresponding to the area of the pigmented macule. This radiopacity was present above the alveolar bone embedded inside the oral mucosa (Figure 1d). Further Energy Dispersive X-Ray (EDX) analysis was carried out using Quanta FEG250 (Oxford Instruments, UK) of the excised tissue. It revealed presence of silver as one of the components (Figure 1e). This helped in confirming that the pigmented lesion was indeed amalgam tattoo. After 2 weeks, patient was called up for review. The postoperative healing was uneventful due to scar tissue formation but there was no tenderness upon palpation of the post-surgical site and patient was able to chew properly without any discomfort with his dentures on and could proceed to fabrication of denture.

\section{Discussion}

Pigmented lesions in the oral cavity can be due to exogenous or endogenous pigments and may be broadly classified into (i) diffuse and bilateral and (ii) focal lesions. Under focal lesions they may be further classified based on their colour into (i) brown, (ii) grey-blue and (iii) red-blue-purple lesions. Focal pigmented lesions which clinically appear as grey are amalgam tattoos and other foreign body tattoos [2] which are due to exogenous pigments. The most common cause of such exogenous focal pigmentation such as in this case is amalgam or graphite incorporation into the mucosa. They may be caused accidentally or iatrogenically. It can occur in the oral cavity due to broken amalgam pieces falling into extraction sockets, usage of amalgam contaminated dental floss, contamination of mucosal abrasions with amalgam dust during restorations, remnants of amalgam trapped in the surgical site following retrofill endodontic procedures, and/or embedding of amalgam particles into the oral mucosa produced by pressure of high-speed dental drills $[1,3]$.

Clinically, amalgam tattoos appear as localized grey, blue or black pigmentation on the oral mucosal tissue with ill- or well-defined borders. They can be diffuse, flat macules or slightly raised lesions. The size can be of varying diameters, usually limited to a few millimeters. Diascopy would usually elicit negative results showing that these lesions do not blanch on pressure. It is reported that the lesion is most commonly seen on alveolar mucosa as in this case and followed by gingiva, palate and buccal mucosa. It is said to be more common in the mandibular region[4] as in this case. They are usually asymptomatic and do not cause any complications. No specific treatment is usually required for amalgam tattoos, with most of them found incidentally [3]. However, in this case they caused tenderness since a large fragment of the metal was incorporated into the mucosa, which, on pressure from

Figure 1A: Intraoral photograph of the pigmented lesion on the lower alveolar mucosa.

Figure 2B: Photograph of the wax block with an embedded tissue and a metal fragment at the center of the tissue (white arrow).

Figure 3C: Photomicrograph of the tissue with a central cleft artifact and numerous pigmentations within the surface epithelium and the connective tissue (original magnification: 100x; stain: H\&E).

Figure 4D: A dental panoramic tomography (DPT) with a radiopacity (black circle) and Figure 5E: EDX analysis show the presence of silver $(\mathrm{Ag})$ as one of the components in the tissue.

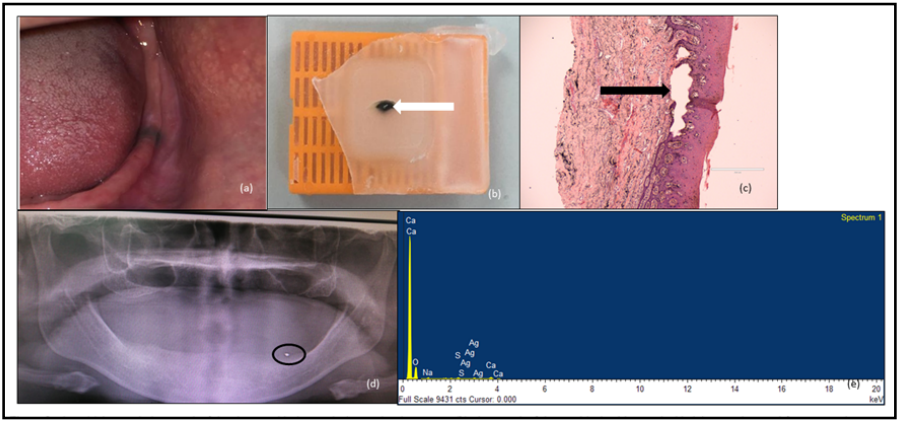


the overlying denture caused tenderness and result edinanill-fittingdenture. Ill-fitting dentures are normally the effect of changes occurring on the soft tissues over time. The short-term symptoms include discomfort, unstable denture, difficulty in speaking, eating and swallowing, pain in the gums, often accompanied with bleeding, a clicking noise when eating, and halitosis or a strange taste in the mouth. In the long run, if this is not managed, the patient may encounter inflammation, ulceration, infection, and in the worst scenario oral cancer. From what has been elicited from the patient's memory, the metal fragment could have been embedded into his tooth socket when the tooth was being extracted. Over time, due to the residual ridge resorption as shown in the DPT (Figure 1d), the fragment could have reached the mucosal level leading to discomfort when in contact with the denture surface. This may also explain why the patient never complained of any discomfort during the tenure of the first pair of dentures when the metal fragments may have been embedded in the hard tissue or deeper in the soft tissue.

As previously mentioned, the cause for the ill-fitting denture in this case was the incorporation of a metal fragment into the mucosa at the pigmented lesion area of the lower alveolar mucosa. However, the incorporation of the metal fragment into the lower alveolar mucosa was missed during clinical examination. Since the patient complained of tenderness at the pigmented lesion area, a biopsy was carried out. After the biopsy while cutting the tissue to prepare for a histopathology slide, the metal fragment embedded within the tissue was noted due to the breakage of the microtome blade. This led to the retrospective review of the DPT of the patient while the histopathology report suggested a diagnosis of amalgamtattoo.

Retrospective review of the DPT resulted in noticing the radiopaque metal fragment embedded in the lower alveolar mucosa at the same place where the patient complained of tenderness on palpation and the presence of the pigmented lesion clinically. Proper clinical examination and radiographic investigation could have avoided such discomfort and agony to the patient. If the clinician had noticed the amalgam fragment embedded in the alveolar mucosa, he could have either referred the patient to remove it before denture construction or relived the denture surface at the pigmented lesional area while constructing the denture. Diagnosis of amalgam tattoo is usually readily determined from the dental history narrated by the patient and clinical examination done in the operatory. However, in this case the patient was not able to remember his previous dental treatment history. This explains well why it is of utmost importance to maintain proper and comprehensive patient records. In the recent past, it has been argued that patient should be given access to their health records. Access to health records appeared to enhance patients' perceptions of control and reduced or had no effect on patient anxiety [5].

The standard investigative methods for differentiating amalgam tattoo from other pigmentation lesions include radiographs [3], infrared spectroscopy [6], reflectance confocal microscopy (RCM) [6], and biopsy [3]. Amalgam tattoos appear radiopaque in radiographs; however, a definitive diagnosis cannot be arrived based on radiography alone $[1,3,7,8]$ as in this case. Infrared spectroscopy has been reported to be a suitable technique for differentiating amalgam tattoo over other melanocytic lesions in formalin-fixed paraffin-embedded tissue sample [9], and it is less invasive compared to biopsy as it does not require any excision procedures.
RCM has been proven to allow imaging with cellular resolution and has excellent diagnostic accuracy to diagnose cutaneous melanoma [10],. The first description of amalgam tattoo using RCM has been carried out [6], but there is limited evidence to prove that it is a reliable test to diagnose amalgam tattoo although its use has been expanded beyond skin to oral and genital mucosa especially for differentiating mucosal melanoma from other benign lesion [11-13].

In this case since the biopsy was already carried out and the histopathology was consistent with amalgam tattoo the above-mentioned methods for the diagnosis were not required. However, to differentiate other foreign body tattoos such as graphite incorporation form amalgam tattoo EDX analysis was carried out which clearly showed the presence of silver in the excised tissue. But we were unable to find any mercury in the EDX analysis. We speculate that this could be due to the leaching of the mercury from the metal fragments which occurs over time. Most importantly, treatment planning is very essential to issue the problem of patient. In this case, the take home message is to design a proper treatment plan prior to any construction of prosthesis with aids of accurate definitive diagnosis.

\section{Conclusion \& Recommendation}

In conclusion, we recommend that asymptomatic amalgam tattoos presenting on the edentulous alveolar ridge must be viewed with caution before fabrication of dentures, partial or complete. The lesions must be palpated to rule out tenderness upon exertion of pressure. It is recommended that a thorough investigation with radiographs or other means of the pigmented lesion be carried out before fabrication of dentures, even if the lesion is clinically apparent to be an amalgam tattoo. If tenderness is present, then it is advised to surgically remove the amalgam tattoo before fabrication of dentures, lest patient non- compliance in wearing the dentures, due to discomfort upon pressure exertion while chewing. This is to avoid development of tenderness or discomfort in the future following the insertion of thedentures.

\section{Acknowledgement}

The authors would like to thank Ms. Helen Ng Lee Ching and Mr. Hassan bin Ismail of Biomaterial Research Laboratory (BRL), Dental Research Management Centre, Faculty of Dentistry, University of Malaya, Kuala Lumpur, Malaysia for helping us with the EDX analysis of the tissue.

\section{References}

[1]. Neville, B.W., et al., Oral and maxillofacial pathology. 2015: Elsevier Health Sciences, Elsevier Publications; 2009. pp. 308-13.

[2]. Kauzman, A., et al., Pigmented lesions of the oral cavity: review, differential diagnosis, and case presentations. J Can Dent Assoc, 2004. 70(10): p. 682-3.

[3]. Buchner, A. and L.S. Hansen, Amalgam pigmentation (amalgam tattoo) of the oral mucosa: A clinicopathologic study of 268 cases. Oral Surgery, Oral Medicine, Oral Pathology, 1980. 49(2): p. 139-147.

[4]. Vera-Sirera, B., et al., Clinicopathological and immunohistochemical study of oral amalgam pigmentation. Acta Otorrinolaringol Esp, 2012. 63(5): p. 376-81.

[5]. Davis Giardina, T., et al., Patient access to medical records and healthcare outcomes: a systematic review. J Am Med Inform Assoc, 2014. 21(4): p. $737-41$.

[6]. Yélamos, O., et al. In vivo intraoral reflectance confocal microscopy of an amalgam tattoo. Dermatology practical \&amp; conceptual, 2017. 7, 13-16 
DOI: $10.5826 /$ dpc.0704a04.

[7]. Buchner, A., Amalgam tattoo (amalgam pigmentation) of the oral mucosa: clinical manifestations, diagnosis and treatment. Refuat Hapeh Vehashinayim (1993), 2004. 21(2): p. 19-22, 96.

[8]. Krahl, D., A. Altenburg, and C.C. Zouboulis, Reactive hyperplasias, precancerous and malignant lesions of the oral mucosa. JDDG: Journal der Deutschen Dermatologischen Gesellschaft, 2008. 6(3): p. 217232.

[9]. Laimer, J., et al., Amalgam tattoo versus melanocytic neoplasm - Differential diagnosis of dark pigmented oral mucosa lesions using infrared spectroscopy. PLoS One, 2018. 13(11): p. e0207026.

[10]. Pellacani, G., et al., The impact of in vivo reflectance confocal microscopy for the diagnostic accuracy of melanoma and equivocal melanocytic lesions. J Invest Dermatol, 2007. 127(12): p. 2759-65.

[11]. Maher, N.G., et al., In vivo reflectance confocal microscopy for evaluating melanoma of the lip and its differential diagnoses. Oral surgery, oral medicine, oral pathology and oral radiology, 2017. 123(1): p. 84-94.

[12]. Uribe, P., et al., In Vivo Reflectance Confocal Microscopy for the Diagnosis of Melanoma and Melanotic Macules of the Lip. JAMA Dermatol, 2017. 153(9): p. 882-891.

[13]. Debarbieux, S., et al., Reflectance confocal microscopy of mucosal pigmented macules: a review of 56 cases including 10 macular melanomas. $\mathrm{Br}$ J Dermatol, 2014. 170(6): p. 1276-84. 